\title{
Application of Real-Time Visual Feedback System in Balance Train- ing of the Center of Pressure with Smart Wearable Devices in women: a randomized controlled trial
}

\section{I-Lin Wang ${ }^{1}$, Li-I Wang ${ }^{2}$, Yang Liu ${ }^{3}$, Shi-Jie Xue ${ }^{3}$, Rui Hu ${ }^{3}$, Yu Su ${ }^{3}$, and Chun-Sheng $\mathrm{HO}^{4}{ }^{*}$}

1 College of Physical Education, Hubei Normal University, Huangshi 435002, Hubei, China, ilin.wang@hotmail.com (I-L.W.)

2 Department of Physical Education and Kinesiology, National Dong Hwa University, Hualien 97046, Taiwan, tennis01@mail.ndhu.edu.tw (L-I. W.)

3 Graduate Institute, Jilin Sport University, Changchun 130022, Jilin, China, 972226173@gmail.com (Y.L.); xue759851324@gmail.com (S-J. X.); ruihu0614@gmail.com (R.H.); y.su825@gmail.com (Y.S.)

4 Department of Physical Therapy, College of Medical and Health Science, Asia University, Taichung, Taiwan Division of Physical Medicine and Rehabilitation, Lo-Hsu Medical Foundation, Inc., Lotung Poh-Ai Hospital, Yilan, Taiwan, cochonho@gmail.com

* Correspondence: cochonho@gmail.com 


\section{Abstract}

This study aims to explore the effect of real-time visual feedback (VF) information of the pressure of center $(\mathrm{COP})$ provided by intelligent insoles on balance training in a one leg stance (OLS) and tandem stance (TS) posture. Thirty healthy female college students were randomly assigned to the visual feedback balance training group (VFT), non-visual feedback balance training group (NVFT), and control group (CG). The balance training includes: OLS, tandem Stance (dominant leg behind, TSDL), tandem stance (non-dominant leg behind, TSNDL). The training lasted 4 weeks, the training lasts 30 minutes at an interval of 1 days. There was a significant difference in the interaction effect between Groups*Times of the COP parameters $(\mathrm{p}<0.05)$ for OLS. There was no significant difference in the interaction effect between Groups*Times of the COP parameters $(\mathrm{p}>0.05)$ for TS. The main effect of the COP parameters was a significant difference in Times $(\mathrm{p}<0.05)$. The COP displacement, velocity, radius, and area in VFT significantly decreased after training $(\mathrm{p}<0.05)$. Therefore, the visual feedback technology of intelligent auxiliary equipment during balance training can enhance the benefit of training. The use of smart wearable devices in OLS balance training may improve the visual and physical balance integration ability.

Key words: balance training, real-time visual feedback, smart wearable devices, center of pressure 


\section{Introduction}

An increase in age is usually accompanies by degeneration in sensory and neuromuscular control mechanisms, thereby causing adverse effects on posture control [1]. Impaired posture control can seriously affect physical function and cause falls in the elderly [2]. Decreased balance function and poor posture control are closely related to an increased risk of falls and mobility difficulties. Balance ability refers to the human body to adjust automatically to maintain postural stability when it moves or is subjected to external forces [3]. Balance control is usually affected by joint range of motion and muscle strength, which can be used to monitor the sensory information of the mechanism [4]. Therefore, good balance must be regulated by the sensory system and neuromuscular system.

During upright posture control, people are clearly aware of their position changes in space when they give VF based on the displacement of the foot center of pressure (COP) or body center of mass (COM) [5]. The visual system can provide the human body with information about the surrounding environment, location, direction, and speed during movement. When the visual information is removed or altered, the action system must compensate by receiving proprioceptive feedback and sensory information from the vestibular system in order to maintain balance [6]. Therefore, VF can help increase the body's stability and balance ability while controlling the posture of the human body. In recent years, sensorimotor integration technology has been used to provide VF to improve the balance ability of people with disabilities and highrisk falls. Previous studies have indicated that internal feedback on one's own posture sway can be obtained through VF so that the body can control its posture changes more autonomously 
[7]. In a study detailing the effect of VF from COP on balance posture control of adolescents and the elderly, it was found that the use of VF for COP in the standing task is a common method for evaluating and training posture control [8]. Therefore, VF can improve the upright posture control and change postural sway in the anterior-posterior and medial-lateral directions to maintain balance. In addition, further findings on ankle movement can clarify different types of VF on body sway and ankle joint mechanisms that contribute to postural sway control [9]. The comparison between the traditional body training and computer vision feedback training indicated that the computer vision feedback group had a better effect on the balance posture control of the human body [10]. Therefore, providing VF in balance training can effectively improve the balancing ability of participants.

VF training to control body posture helps improve the body's ability to maintain balance and achieve a stable standing effect. It can stabilize the body posture and significantly improve static and dynamic balance ability [11]. Previous studies have found that COP displacement and mean velocity of patients with spinal cord injury decreased after VF standing balance training, indicating that the ability of static and dynamic stability improved significantly after training [11]. After applying wearable devices to balance training for the elderly, the COP area and COP parameters displayed a significantly decrease, indicating that balance training is effective for improving postural control and functional performance in older adults [12]. These studies used screen COP displacement projection onto the screen as balance training to maintain stability. Therefore, appropriate external real-time VF information (the position of the real-time COP) should be provided during balance training to improve the control ability of posture balance and increase the benefit of training. 
In summary, effectively using the real-time VF information of COP provided by smart wearable devices for balance control and training can benefit technology-assisted balance training at home, thereby aiding sports training and physical rehabilitation. This study aims to enhance the benefit of balance training effectively by using the real-time VF information of COP provided by smart insoles in the OLS or TS posture. This study demonstrates the effect of a simple technology-assisted VF system on body balance.

\section{Materials and Methods}

\section{1. participants}

Thirty healthy female college students were recruited and randomly assigned to the VFT, NVFT, and CG with 10 persons in each group. The height $(167.59 \pm 4.68 \mathrm{~cm})$, weight $(57$. $10 \pm 7.15 \mathrm{~kg})$, and age $(20.12 \pm 1.13)$ of each participant were recorded. None of the participants had any known neurological, motor, visual impairment, or disability. The participants were informed of the content, process, and precautions for the study group. The test instructions were read out to them and they understood and were willing to cooperate fully with the experimenter and signed the consent form. The study was approved by the Research Ethics Committee of Hualien Tzu Chi Hospital, Buddhist Tzu Chi Medical Foundation (IRB109-053-B) and was conducted in accordance with the Declaration of Helsinki.

\subsection{Equipment}

A force plate (BTS P6000, BTS Bioengineering, Italy) was used to calculate the coordinates of the COP displacement in the mediolateral (ML) and anteroposterior (AP) directions and the 
COP velocity. Force plate signals were collected at a sampling frequency of $300 \mathrm{~Hz}$ and synchronized with the motion analysis system. In order to avoid the impact of different wear and tear during the test and training, all participants wore the same experimental tights and uniform sports shoes. The training shoes were equipped with the same intelligent leg pad as the VF training group to avoid the interference from the insole material, which could affect the training effect. The use of iPad Pro with Podoon APP was simultaneously studied as a VF device.

\subsection{Procedures}

Participants were recruited prior to the experiment and their foot length was measured. Then, the smart foot pad matching their foot length was selected and cut. The participants would have a five-minute warm-up run and one minute of rest. After this preparation, thirty female college students were randomly divided into three groups: VFT, NVFT, and CG without any training. The training includes OLS-NF, OLS-VF, TSDL-NF, TSDL-VF, TSNDL-NF, and TSNDL-VF $[13,14]$. It lasted 4 weeks, and took place thrice a week. It comprised of balance training for 30 min each (static standing for $30 \mathrm{~s}$ ) at intervals of 1-2 days. In OLS, participants were instructed to stand on their dominant leg, while the non-supported leg was flexed at the knee with the plantar surface of the foot stabilized on the knee of the supporting leg [15]. In TS, the participants ' feet (on a line, heel-toe position) were placed on the center of the force plate [16]. They were asked to keep the dynamic point in the central circle as much as possible. The iPad Pro was located at an eye-level height, $1 \mathrm{~m}$ apart from the participants. After 4 weeks, the content of the post-test was found to be the same as that of the pretest. 


\subsection{Statistical analysis}

In this study, the average values of three test results in each subject's test action were calculated and used for statistical analysis. MATLAB (R2014a, The MathWorks, USA) was used for statistical analysis. The experiment used mixed design two-way analysis of variance (ANOVA) (Group $\times$ Times) to compare the differences in pretest and post-test among the VFT, NVFT, and CG. For each measurement, post hoc least significant difference (LSD) comparisons were performed on the significant effects. The level of significance was set at $\alpha<0.05$.

\section{Results}

In this study, participants were divided into the VFT, NVFT, and CG under VF and NF conditions for 4 weeks. The training results of the COP ML max displacement or COP AP max displacement are depicted in Figure 1. In OLS-NF, there was a significant difference in the interaction effect between Groups*Times $(p<0.001)$, the simple main effect of the COP ML max displacement showed a significant difference among the three groups in the post-test $(\mathrm{p}=0$. 003), post hoc analysis showed a significant decrease in VFT/CG and NVFT/CG ( $\nabla 36.87 \%$, $\mathrm{p}=0.001, \nabla 18.08 \%, \mathrm{p}=0.031), \mathrm{VFT}$ and NVFT were significantly decreased after training $(\nabla 18.38 \%, \mathrm{p}<0.001$ and $\nabla 2.99 \%, \mathrm{p}=0.001)$. The simple main effect of the COP AP $\max$ displacement showed a significant difference among the three groups in the post-test $(\mathrm{p}=0$. 044), post hoc analysis showed a significant decrease in VFT/CG and NVFT/CG ( $\nabla 33.01 \%$, $\mathrm{p}=0.030$ and $\nabla 33.35 \%, \mathrm{p}=0.029)$, and VFT was significantly decreased after training $(\nabla 31$. $94 \%, p=0.018)$. In OLS-VF, there was a significant difference in the interaction effect between Groups*Times $(\mathrm{p}=0.031)$, the simple main effect of the COP ML max displacement showed a 
significant difference among the three groups in the post-test $(p=0.010)$, post hoc analysis showed significant decrease in VFT/CG, NVFT/CG ( $\nabla 35.99 \%, \mathrm{p}=0.009$ and $\nabla 37.44 \%$, $\mathrm{p}=0.007)$, VFT was significantly decreased after training $(\nabla 21.76 \%, \mathrm{p}=0.005)$. The simple main effect of the COP AP max displacement showed significant differences among the three groups in the post-test $(\mathrm{p}<0.001)$, post hoc analysis showed a significant decrease in VFT/ NVFT ( $\nabla 87.07 \%, \mathrm{p}<0.001)$, VFT/CG and NVFT/CG were significantly decreased $(\nabla 123$. $00 \%, \mathrm{p}<0.001$ and $\nabla 19.21 \%, \mathrm{p}<0.001)$, VFT was significantly decreased after training (54. $22 \%, \mathrm{p}=0.008)$.

In TSNDL-NF, there was no significant difference in the interaction effect between Groups*Times of COP ML max displacement and COP AP max displacement $(\mathrm{p}=0.368$ and $\mathrm{p}=0$. 325). The main effect of the COP ML max displacement was a significant difference between the groups $(\mathrm{p}<0.001)$, analysis of pretest and post-test showed significant decrease in VFT and NVFT $(\nabla 20.44 \%, \mathrm{p}=0.005$ and $\nabla 21.74 \%, \mathrm{p}=0.014)$. The main effect of the COP AP max displacement showed a significant difference between the groups $(p=0.030)$, analysis of pretest and post-test showed a significant decrease in VFT $(\nabla 26.74 \%, \mathrm{p}=0.002)$. In TSDL-NF, there was no significant difference in the interaction effect between Groups*Times of COP ML max displacement and COP AP max displacement $(\mathrm{p}=0.151$ and $\mathrm{p}=0.135)$. The main effect of the COP ML max displacement was a significant difference between the groups $(\mathrm{p}=0.004)$, analysis of pretest and post-test showed a significant decrease in VFT ( $\nabla 29.97 \%, \mathrm{p}=0.003)$. The main effect of the COP AP max displacement was a significant difference between the groups $(\mathrm{p}=0.047)$, analysis of pretest and post-test showed a significant decrease in VFT $(\nabla 24.15 \%, \mathrm{p}=0.031)$.In TSNDL-VF, there was no significant 
difference in the interaction effect between Groups*Times of COP ML max displacement and COP AP max displacement ( $p=0.123$ and $p=0.273$ ). The main effect of the COP ML max displacement was a significant difference between the groups $(\mathrm{p}=0.006)$, analysis of pretest and post-test showed a significant decrease in VFT $(\nabla 12.75 \%, \mathrm{p}=0.025)$. The main effect of the COP AP max displacement was a significant difference between the groups $(p=0.003)$, analysis of pretest and post-test showed a significant decreased in $\operatorname{VFT}(\nabla 31.08 \%, \mathrm{p}<0.001)$. In TSDL-VF, there was no significant difference in the interaction effect between Groups*Times of COP ML max displacement and COP AP max displacement ( $p=0.079$ and $\mathrm{p}=0$. 063). The main effect of the COP ML max displacement was a significant difference between the groups ( $\mathrm{p}=0.009)$, analysis of pretest and post-test showed a significant decrease in VFT or NVFT $(\nabla 43.12 \%, \mathrm{p}<0.001$ and $\nabla 46.88 \%, \mathrm{p}<0.001)$. The main effect of the COP AP max displacement was a significant difference between the groups ( $\mathrm{p}=0.004)$, analysis of pretest and post-test showed a significant decrease in VFT $(\nabla 18.27 \%, \mathrm{p}=0.001)$. Therefore, compared with the NVFT and CG, the COP ML max displacement and COP AP max displacement in the VFT were significantly decreased, which proved that VFT was beneficial in improving the balance ability of the human body. 

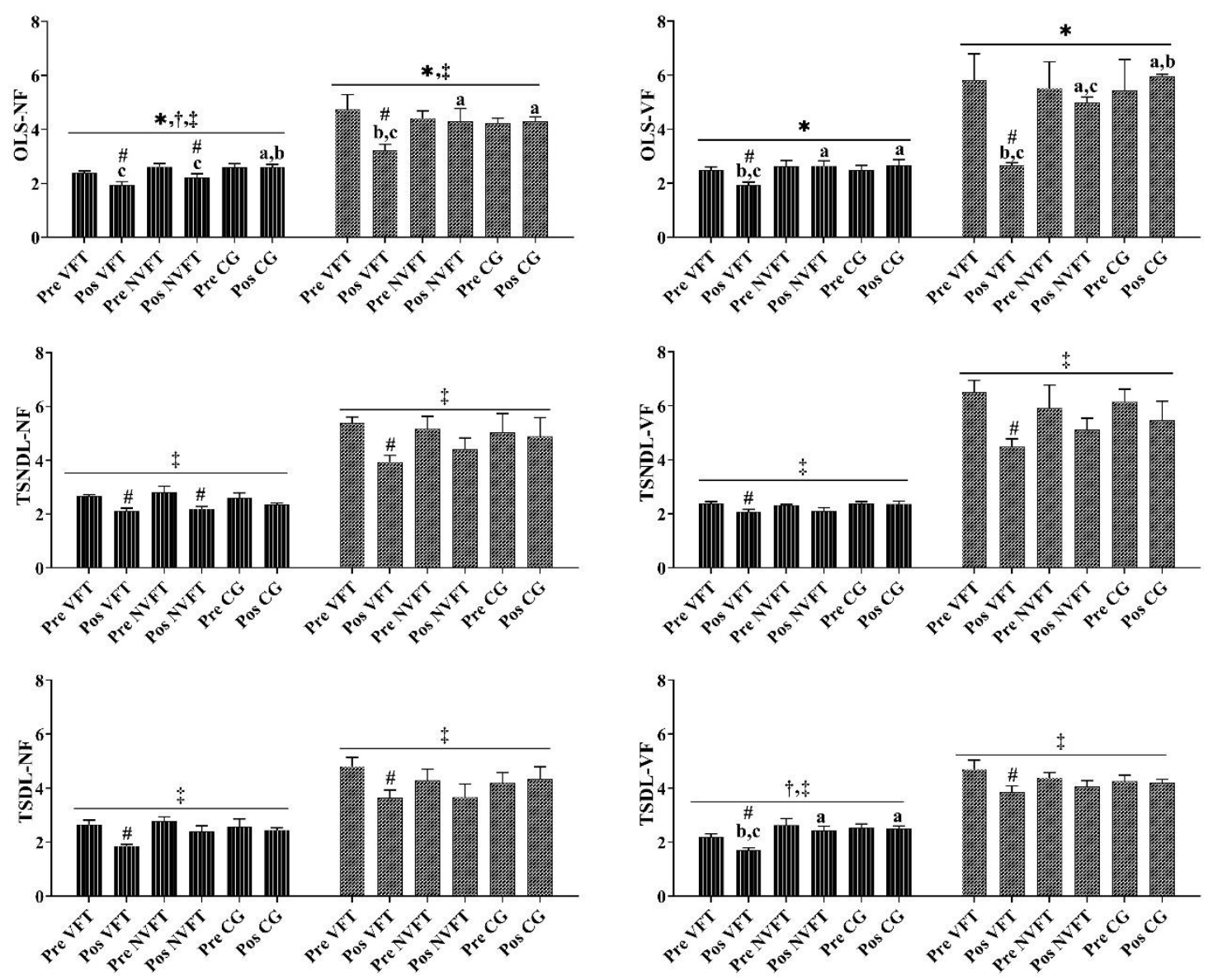

Figure 1. Mean + SD of Times: pre and post training * Group: VFT 、 NVFT and CG for the OLS-NF, OLS-VF, TSDL-NF, TSDL-VF, TSNDL-NF, and TSNDL-VF in COP ML and COP AP max displacement parameter. indicates a significant difference in interaction (Group*Times) $(\mathrm{p}<0.05) .{ }^{\dagger}$ represents a significant difference in the main effect (group) $(\mathrm{p}<0.05) .{ }^{*}$ indicates a significant difference in the main effect $($ Times $)(\mathrm{p}<0.05) .{ }^{\#}$ indicates a significant difference from pretest. ${ }^{\mathrm{a}}$ indicates a significant difference with VFT. ${ }^{\mathrm{b}}$ indicates a significant difference with NVFT. ${ }^{c}$ indicates a significant difference with CG. There was a significant difference in the interaction (Group*Times) of the COP ML max displacement or COP AP max displacement in OLS ( $<<0.05$ ). 
AP max displacement in TS ( $\mathrm{p}>0.05)$.

The training results of the COP ML velocity or COP AP velocity are listed in Figure 2. In OLS-NF, there was a significant difference in the interaction effect between Groups*Times $(\mathrm{p}=0.013)$, the simple main effect of the COP ML velocity showed a significant difference among the three groups in the post-test $(\mathrm{p}<0.001)$, post hoc analysis showed a significant decrease in VFT/CG and NVFT/CG $(\nabla 22.89 \%, \mathrm{p}<0.001$ and $\nabla 14.21 \%, \mathrm{p}=0.006), \mathrm{VFT}$ significantly decreased after training $(\nabla 6.30 \%, \mathrm{p}=0.003)$. The simple main effect of the COP AP velocity showed a significant difference among the three groups in the post-test $(\mathrm{p}=0.002)$, post hoc analysis showed a significant decrease in VFT, NVFT, and CG $(\nabla 28.32 \%, \mathrm{p}=0.001)$, VFT significantly decreased after training $(\nabla 7.30 \%, \mathrm{p}=0.009)$.In OLS-VF, there was a significant difference in the interaction effect between Groups*Times $(\mathrm{p}=0.018)$, the simple main effect of the COP ML velocity showed a significant difference among the three groups in the post-test $(\mathrm{p}<0.001)$, post hoc analysis showed a significant decrease in VFT/CG, NVFT/CG $(\nabla 30.85 \%, \mathrm{p}<0.001$ and $\nabla 24 \%, \mathrm{p}<0.001)$, VFT and NVFT were significantly decreased after training $(\nabla 19.94 \%, \mathrm{p}=0.001$ and $\nabla 17.93 \%, \mathrm{p}=0.003)$. The simple main effect of the COP AP velocity showed a significant difference among the three groups in the post-test $(\mathrm{p}<0$. 001), post hoc analysis showed a significant decrease in VFT/CG and NVFT/CG ( $\nabla 22.44 \%$, $\mathrm{p}<0.001, \nabla 13.74 \%, \mathrm{p}=0.002)$, VFT significantly decreased after training $(\nabla 17.10 \%, \mathrm{p}=0$. 001, $\nabla 8.40 \%, \mathrm{p}=0.031)$.

In TSNDL-NF, there was no significant difference in the interaction effect between Groups*Times of COP ML velocity or COP AP velocity ( $\mathrm{p}=0.570$ and $\mathrm{p}=0.170)$. The COP 
ML velocity was significantly different between the groups $(p=0.038)$, analysis of pretest and post-test showed a significant decrease in VFT/CG and NVFT/CG $(\nabla 9.42 \%, \mathrm{p}=0.009$ and $\nabla$ $12.20 \%, \mathrm{p}=0.001)$. VFT and NVFT were significantly decreased in training $(8.69 \%, \mathrm{p}<0$. 001). The main effect of the COP AP velocity was a significant difference between the groups $(\mathrm{p}=0.043)$, analysis of pretest and post-test displayed a significant decrease in VFT $(\nabla 13$. $61 \%, p=0.003)$. In TSDL-NF, there was no significant difference in the interaction effect between Groups*Times of COP ML velocity or COP AP velocity $(p=0.598$ and $p=0.598)$. The COP ML velocity was significantly different between the groups $(p=0.049)$, analysis of pretest and post-test illustrated a significant decrease in VFT $(\nabla 3.50 \%, \mathrm{p}=0.009)$. The main effect of the COP AP velocity was a significant difference between the groups ( $p=0.049$ ), analysis of pretest and post-test showed a significant decrease in VFT $(\nabla 11.35 \%, p=0.004)$. In TSNDL-VF, there was no significant difference in the interaction effect between Groups*Times of COP ML velocity or COP AP velocity ( $p=0.080$ and $p=0.106)$. The COP ML velocity was not significantly different between the groups $(p=0.493)$, there was a significant difference in time ( $\mathrm{p}=0.034)$, analysis of pretest and post-test showed a significant decrease in VFT $(\nabla 3.20 \%, \mathrm{p}=0.037)$. The main effect of the COP AP velocity was a significant difference between the groups $(\mathrm{p}=0.025)$, analysis of pretest and post-test showed a significant decrease in VFT ( $\nabla 7.50 \%, \mathrm{p}=0.047)$. In TSDL-VF, there was no significant difference in the interaction effect between Groups*Times of COP ML velocity or COP AP velocity $(p=0.228$ and $\mathrm{p}=0.808)$, the COP ML velocity was not significantly different between the groups $(\mathrm{p}=0$. 022 ), analysis of pretest and post-test showed a significant decrease in VFT ( $\nabla 6.60 \%, \mathrm{p}<0$. 001). The main effect of the COP AP velocity was a significant difference between the groups 
$(\mathrm{p}=0.011)$, analysis of pretest and post-test indicated a significant decrease in VFT $(\nabla 6.60 \%$, $\mathrm{p}=0.002$ ). Therefore, compared with the NVFT and CG, there was a significant decrease in the COP ML velocity or COP AP velocity in the VFT, which proved that the VFT was superior to the NVFT and CG.

COP ML velocity $(\mathrm{cm} / \mathrm{s})$ $\mathbb{N}$ COP AP velocity $(\mathrm{cm} / \mathrm{s})$
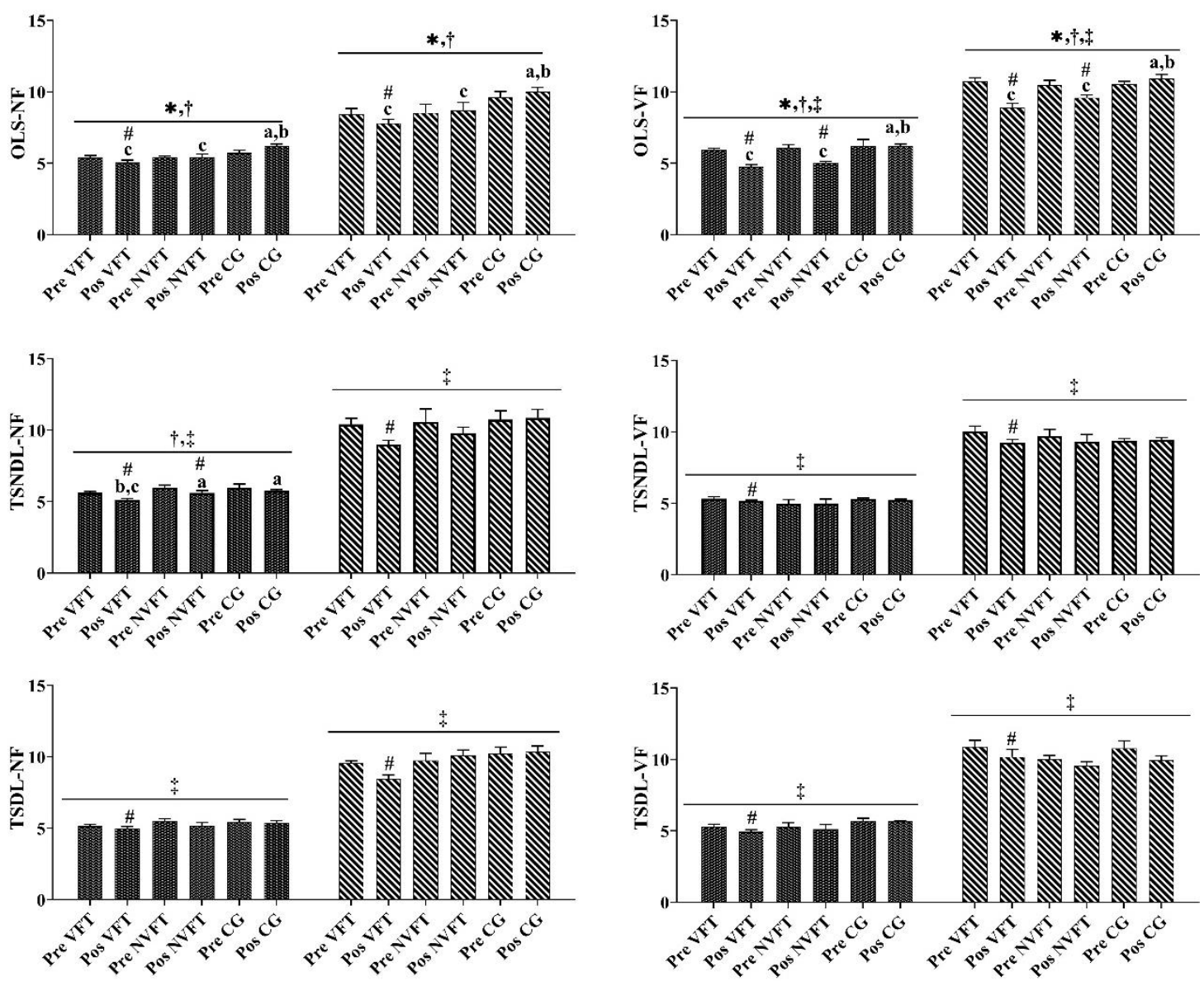

Figure 2. Mean + SD of Times: pre and post training * Group: VFT $、$ NVFT and CG for the OLS-NF, OLS-VF,

TSDL-NF, TSDL-VF, TSNDL-NF, and TSNDL-VF in COP ML and COP AP velocity parameter. ${ }^{*}$ indicates a significant difference in interaction (Group*Times) $(\mathrm{p}<0.05) .{ }^{\dagger}$ represents a significant difference in the main 
effect (group) $(\mathrm{p}<0.05) .{ }^{\dagger}$ indicates a significant difference in the main effect (Times) $(\mathrm{p}<0.05) .{ }^{\#}$ indicates a significant difference from pretest. ${ }^{\mathrm{a}}$ indicates a significant difference with VFT. ${ }^{\mathrm{b}}$ indicates a significant difference with NVFT. ${ }^{\mathrm{c}}$ indicates a significant difference with CG. There was a significant difference in the interaction (Group*Times) of the COP ML velocity or COP AP velocity in OLS $(\mathrm{p}<0.05)$. There was no significant difference in the interaction (Group*Times) of the COP ML velocity or COP AP velocity in TS ( $>>0.05$ ).

The training results of the COP radius and COP area are presented in Figure 3. In OLS-NF, there was a significant difference in the interaction effect between Groups*Times $(p=0.001)$. The simple main effect of the COP radius showed significant differences among the three groups in the post-test $(\mathrm{p}=0.005)$, post hoc analysis displayed a significant decrease in VFT/CG and NVFT/CG $(\nabla 34.34 \%, \mathrm{p}=0.031$ and $\nabla 53.77 \%, \mathrm{p}=0.001)$, and the VFT significantly decreased after training $(\nabla 28.67 \%, \mathrm{p}=0.001)$. The simple main effect of the COP area showed significant differences among the three groups in the post-test $(\mathrm{p}=0.016)$, post hoc analysis showed a significant decrease in VFT/CG and NVFT/CG ( $\nabla 64.90 \%, \mathrm{p}=0.047, \nabla 95.39 \%$, $\mathrm{p}=0.005)$, VFT significantly decreased after training $(\nabla 42.83 \%, \mathrm{p}<0.001)$. In OLS-VF, there was a significant difference in the interaction effect between Groups*Times $(p=0.002)$, the simple main effect of the COP radius showed significant differences among the three groups in the post-test $(\mathrm{p}=0.002)$, post hoc analysis showed a significant decrease in VFT/CG and NVFT/CG $(\nabla 48.25 \%, \mathrm{p}=0.014$ and $\nabla 69.75 \%, \mathrm{p}=0.001)$, VFT significantly decreased after training $(\nabla 69.75 \%, \mathrm{p}=0.003)$. The simple main effect of the COP area showed significant differences among the three groups in the post-test $(p=0.001)$, post hoc analysis showed a significant decrease in VFT/CG and NVFT/CG $(\nabla 80.54 \%, \mathrm{p}=0.001$ and $\nabla 98.37 \%, \mathrm{p}<$ 0. 001), VFT significantly decreased after training ( $\nabla 47.16 \%, \mathrm{p}<0.001)$. 
In TSNDL-NF, there was no significant difference in the interaction effect between Groups*Times of COP radius and COP area $(p=0.323$ and $p=0.175)$. The main effect of the COP radius was a significant difference between the groups $(\mathrm{p}=0.009)$, analysis of pretest and post-test displayed a significant decrease in VFT $(\nabla 25.76 \%, \mathrm{p}=0.002)$. The main effect of the COP area was a significant difference between the groups $(\mathrm{p}=0.045)$, analysis of pretest and post-test revealed a significant decrease in VFT $(\nabla 35.51 \%, \mathrm{p}=0.022)$. In TSDL-NF, there was no significant difference in the interaction effect between Groups*Times of COP radius and COP area $(p=0.077$ and $p=0.300)$. The main effect of the COP radius was a significant difference between the groups $(\mathrm{p}=0.001)$, analysis of pretest and post-test showed a significant decrease in VFT $(\nabla 19.17 \%, \mathrm{p}<0.001)$. The main effect of the COP area was a significant difference between the groups $(\mathrm{p}=0.045)$, analysis of pretest and post-test revealed a significant decrease in VFT $(\nabla 33.35 \%, \mathrm{p}<0.001)$. In TSNDL-VF, there was no significant difference in the interaction effect between Groups*Times of COP radius and COP area $(p=0.568$ and $\mathrm{p}=0.861$ ). The main effect of the COP radius was a significant difference between the groups ( $\mathrm{p}<0.001)$, analysis of pretest and post-test depicted a significant decreased in VFT and NVFT $(\nabla 16.71 \%, \mathrm{p}<0.001, \nabla 29.14 \%, \mathrm{p}<0.001)$. The main effect of the COP area was a significant difference between the groups ( $\mathrm{p}<0.001)$, analysis of pretest and post-test showed a significant decrease in VFT and NVFT $(\nabla 44.75 \%, \mathrm{p}<0.001, \nabla 31.62 \%, \mathrm{p}<0$. 001). In TSDL-VF, there was no significant difference in the interaction effect between Groups*Times of COP radius and COP area $(\mathrm{p}=0.389$ and $\mathrm{p}=0.052)$. The main effect of the COP radius was a significant difference between the groups $(\mathrm{p}=0.031)$, analysis of pretest and post-test exhibited a significant decrease in VFT $(\nabla 21.31 \%, \mathrm{p}=0.005)$. The main effect of the 
COP area was a significant difference between the groups ( $\mathrm{p}=0.002)$, analysis of pretest and post-test showed a significant decreased in VFT $(\nabla 39.48 \%, \mathrm{p}<0.001)$. Therefore, the COP radius and COP area in the VFT decreased significantly, compared with the NVFT and CG, which proves that VFT can enhance the balance control ability of the human body.

COP radius $(\mathrm{cm})$ COP area $\left(\mathrm{mm}^{2}\right)$
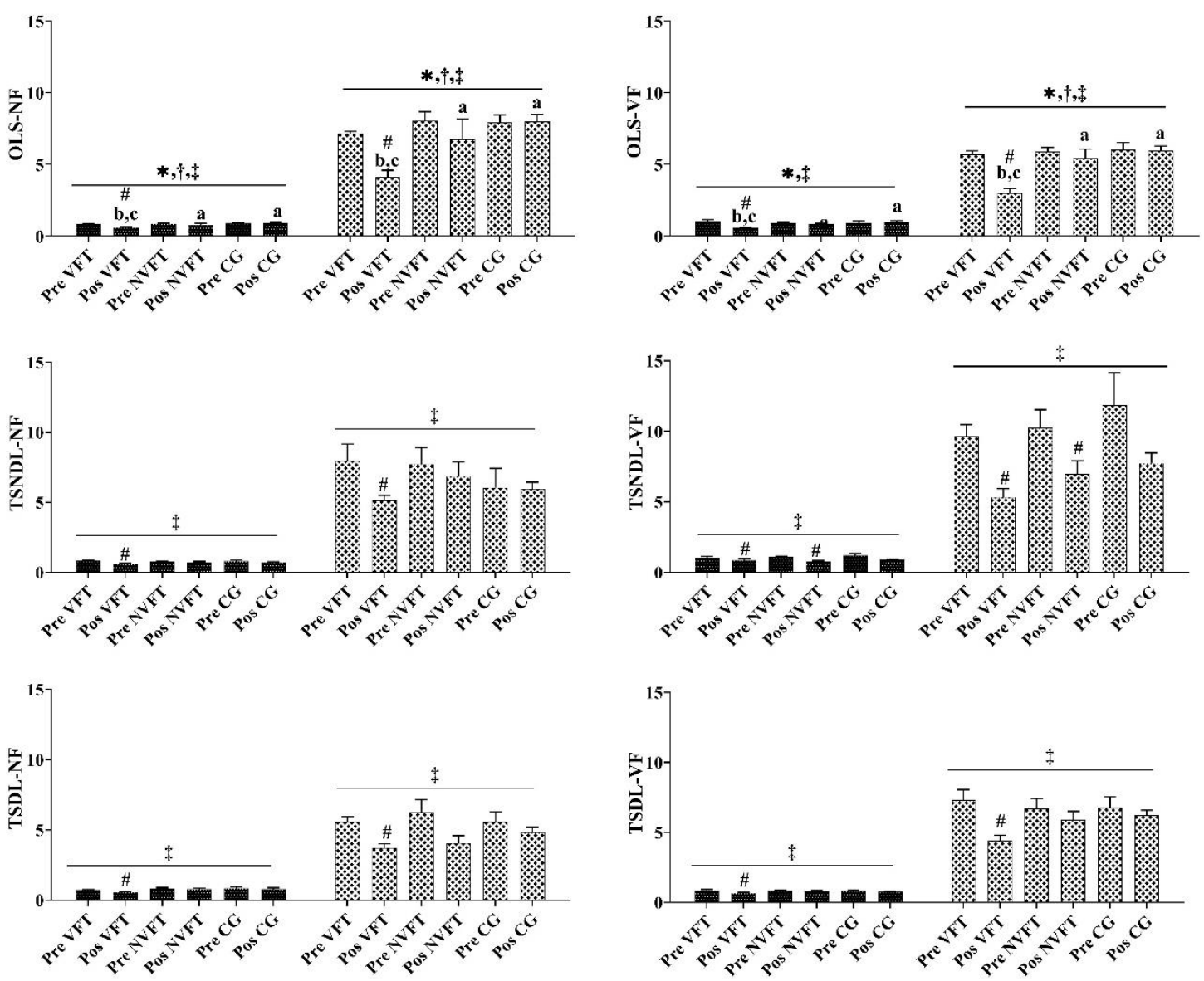

Figure 3. Mean + SD of Times: pre and post * Group: VFT - NVFT and CG for the OLS-NF, OLS-VF, TSDLNF, TSDL-VF, TSNDL-NF, and TSNDL-VF in COP radius and COP area parameter. ${ }^{*}$ indicates a significant difference in interaction (Group*Times) $(\mathrm{p}<0.05) .{ }^{\dagger}$ represents a significant difference in the main effect (group) 
$(\mathrm{p}<0.05) .+$ indicates a significant difference in the main effect $($ Times $)(p<0.05) .{ }^{\#}$ indicates a significant difference from pretest. ${ }^{\mathrm{a}}$ indicates a significant difference with VFT. ${ }^{\mathrm{b}}$ indicates a significant difference with NVFT. ${ }^{\mathrm{c}}$ indicates a significant difference with CG. There was a significant difference in the interaction (Group*Times) of the COP radius or COP area in OLS $(\mathrm{p}<0.05)$. There was no significant difference in the interaction (Group*Times) of the COP radius or COP area in TS $(\mathrm{p}>0.05)$.

\section{Discussion}

The purpose of this study was to perform balance training for participants with the VF technique provided by a smart wearable device of COP, in order to observe the effect of this technique on the static balance posture control of women. After four weeks of balance training, the results showed that visual feedback training can improve the stability of human posture control by OLS and TS static balance training on VFT intelligent App.

In this study, the decrease in COP ML/COP AP displacement and COP ML/COP AP variance in VFT demonstrated that the participants could control body sway in a considerably stable manner with the help of real-time VF information. The body integrates vision, vestibular sense, and somatosensory through the central nervous system (CNS) to maintain human balance performance [17]. After the balance training of external VF, the results of COP ML /COP AP displacement decreased in OLS with participants' dominant/non-dominant leg illustrating that the use of technology-assisted App to provide VF training can help reduce the displacement in the AP and ML directions. In addition, the sway and posture changes in the AP direction are closely related to ankle neuromuscular function. The "ankle strategy" can improve 
ankle stability and reduce COP displacement to improve balance ability [18]. The "ankle strategy" can maintain the body balance in AP direction showing the static posture balance of standing. In this study, the decrease in AP and ML displacement of the COP indicated that the technique-assisted training may increase the balance control ability of the ankle joint to reduce body sway and displacement variation after the participation of VF.

In OLS, the parameters of COP mean velocity, COP ML velocity, and COP AP velocity of VFT after four weeks of training; the NVFT was not different before and after training in the NF test, but there was a difference in the VF test, and the CG remained unchanged. Meanwhile, the COP mean velocity, COP ML velocity, and COP AP velocity in VFT were significantly lower than those in the NVFT and CG. In TS, the COP mean velocity, COP ML velocity, and COP AP velocity in VFT decreased after four weeks of training, and there was no difference between the NVFT and CG. Previous studies have found that the smaller the displacement velocity, the better the balance control ability when using VF training [19]. In this study, VF training using smart auxiliary equipment may help participants maintain better physical stability. When the human body performs visual feedback training, the central nervous system controls the body's goal-directed movements through relevant mechanisms [20]. Posture sway in the ML direction is controlled by adduction/abduction of the hip joint mechanism, while the postural sway in the AP direction is controlled by plantar flexion/dorsiflexion of the ankle joint mechanism [21]. Therefore, in this study, the decrease in COP ML velocity and COP AP velocity in the VFT may be caused by the goal-directed movement of the ankle and hip joint mechanism regulated by the CNS during VF training. Past studies have found that balance training stimulates proprioception and increases sensory motor nerve signal transmission to 
improve balance control ability [22], and balance training will strengthen muscular activity and improve the stability of the balance mechanism [23]. Therefore, training without the assistance of smart devices will strengthen muscle activity, and the central nervous system will mobilize the relevant muscle groups for goal-directed movements during the VF test to improve balance. However, the VFT conducts visual feedback training during the training process and the CNS controls the relevant muscle groups to perform goal-directed movements during training, so that the training effect of the visual feedback training group is higher than that of the general training group. Therefore, the CNS mobilizes more motor neurons to increase the physical stability when performing VF training in OLS and TS.

In OLS, the parameters of COP area, COP radius, and COP radius variance of VFT decreased after four weeks of training; the NVFT and CG remained unchanged. Meanwhile, the COP area, COP radius, and COP variance in the VFT were significantly lower than those of the NVFT and CG. The results demonstrate that using smart wearable auxiliary VF for training has better balance ability than not wearing smart wearable auxiliary training or remaining untrained. Previous studies have shown that the COP radius and the COP area can reflect the static stability of the human body in the process of OLS; the larger the COP area and COP radius, the worse the stability [24]. Therefore, balance training with visual feedback assisted by smart insoles can help participants maintain physical stability. The decrease in COP radius and COP area is primarily due to conscious control by the human body based on the visual information obtained from VF [25]. During the training process, the participants could integrate VF information and motor sensory information to maintain physical stability under the control of the CNS [26]. Previous studies have found that training with VF provided by smart 
devices can improve balance ability. For example, training with VF provided by a balancer (Pro-kin) or balance board (Wii Fit) can reduce the COP radius and COP area of participants and increase the physical stability after training [27]. Therefore, the decrease in COP area, COP radius, and COP radius variance after training in VF provided by smart insoles may also be due to the increase in visual information. In TS, the COP area, COP radius, and COP radius variance of the VFT decreased after four weeks of training, and there was no difference between the NVFT and CG. Consistent with the results of the VFT in OLS, it was observed that physical stability in TS also increased after VF training. In addition, previous studies have pointed out that smart wearable devices are VF to the body's COM, and the COM VF will strengthen autonomous control and reduce posture sway, thereby achieving more efficient posture control or improving balance [28]. The training without smart auxiliary equipment only adjusts itself under the original sense organ system, and cannot judge the position effectively through the VF [29]. Therefore, the balance ability of the NVFT cannot be significantly improved, and the use of VF assisted by smart insoles for training will provide more VF information to strengthen the physical autonomous control ability and improve physical balance.

\section{Conclusions}

The balancing ability of the body can be enhanced by assisting proprioception through the VF system. The balance mechanism is more dependent on visual feedback as the difficulty of the balancing task increases. Whether in the OLS or TS posture, the application of wearable technology to VF balance training can significantly improve the ability of young people to maintain physical stability and adjust in response to physical instability. Obtaining the real- 
time VF of COP in time compensates for the lack of direct observation of plantar pressure in traditional balance training, and is intuitive and simple, provides real-time feedback, and has strong operability. In the future, it could be applied to the simple balance training of different groups to improve visual and physical balance integration.

The smart insoles monitoring system can be applied to different movement states of the human body. A limitation of the study in our investigation on smart insoles has only considered the training of static stance in the OLS and TS postures. In the future, the training benefits of smart insoles can be further explored through gait experiments to examine changes in muscle activity and biomechanics due to the long-term use of smart wearable devices.

Authors' contributions: Conceptualization, I.-L.W. and Y.-M. C.; Methodology, R.H. and Y.L.; Formal analysis, I.-L. W., S.-J. X. and Y.L.; Data curation; S.-J. X. and Y.L.; Writingoriginal draft preparation, S.-J. X., Y.L., J.-Q. L., R.H. and Y.S.; Writing-Review and editing, I.-L. W.; Supervision, I.-L. W. All authors have read and agreed to the published version of the manuscript.

Funding: This research received grant from the Lo-Hsu Medical Foundation, Inc., Lotung PohAi Hospital (Number: E157).

Acknowledgements: This study was supported by the Lo-Hsu Medical Foundation, Inc., Lotung Poh-Ai Hospital (number: E15). The authors have no conflicts of interest to declare.

Conflicts of Interest: The authors declare no conflict of interest.

\section{References}

1. Laughton, C. A.; Slavin, M.; Katdare, K.; Nolan, L.; Bean, J. F.; Kerrigan, D. C.; Phillips, E.; 
Lipsitz, L. A.; Collins, J. J., Aging, muscle activity, and balance control: physiologic changes associated with balance impairment. Gait \& posture. 2003, 18, 101-108.

2. $\quad$ Delbaere, K.; Close, J. C.; Heim, J.; Sachdev, P. S.; Brodaty, H.; Slavin, M. J.; Kochan, N. A.; Lord, S. R., A multifactorial approach to understanding fall risk in older people. Journal of the American Geriatrics Society. 2010, 58, 1679-1685.

3. Wang, J.; Xu, J.; An, R., Effectiveness of backward walking training on balance performance: A systematic review and meta-analysis. Gait Posture. 2019, 68, 466-475.

4. Horak, F.; Kuo, A., Postural adaptation for altered environments, tasks, and intentions. In Biomechanics and neural control of posture and movement, Springer: 2000; pp 267-281.

5. de Brito Silva, P.; Oliveira, A. S.; Mrachacz-Kersting, N.; Laessoe, U.; Kersting, U. G., Strategies for equilibrium maintenance during single leg standing on a wobble board. Gait \& posture. 2016, 44, 149-154.

6. Shubert, T. E., Evidence-based exercise prescription for balance and falls prevention: a current review of the literature. Journal of geriatric physical therapy. 2011, 34, 100-108.

7. Wulf, G., Attentional focus and motor learning: a review of 15 years. International Review of Sport and Exercise Psychology. 2013, 6, 77-104.

8. Alfieri, F. M.; Riberto, M.; Gatz, L. S.; Ribeiro, C. P. C.; Lopes, J. A. F.; Battistella, L. R., Comparison of multisensory and strength training for postural control in the elderly. Clinical interventions in aging. 2012, 7, 119.

9. Freitas, S. M. S. F.; Duarte, M., Joint coordination in young and older adults during quiet stance: effect of visual feedback of the center of pressure. Gait \& posture. 2012, 35, 83-87.

10. Sungkarat, S.; Fisher, B. E.; Kovindha, A., Efficacy of an insole shoe wedge and augmented pressure sensor for gait training in individuals with stroke: a randomized controlled trial. Clinical rehabilitation. 2011, 25, 360-369.

11. Sayenko, D. G.; Alekhina, M. I.; Masani, K.; Vette, A.; Obata, H.; Popovic, M.; Nakazawa, K., Positive effect of balance training with visual feedback on standing balance abilities in people with incomplete spinal cord injury. Spinal cord. 2010, 48, 886-893.

12. Schwenk, M.; Grewal, G. S.; Honarvar, B.; Schwenk, S.; Mohler, J.; Khalsa, D. S.; Najafi, B., Interactive balance training integrating sensor-based visual feedback of movement performance: a pilot study in older adults. Journal of neuroengineering and rehabilitation. 2014, 11, 164.

13. Giagazoglou, P.; Amiridis, I.; Zafeiridis, A.; Thimara, M.; Kouvelioti, V.; Kellis, E., Static balance control and lower limb strength in blind and sighted women. European journal of applied physiology. 2009, 107, 571-9.

14. Roth, A. E.; Miller, M. G.; Ricard, M.; Ritenour, D.; Chapman, B. L., Comparisons of static and dynamic balance following training in aquatic and land environments. Journal of Sport Rehabilitation. 2006, 15, 299-311.

15. Takeda, K.; Mani, H.; Hasegawa, N.; Sato, Y.; Tanaka, S.; Maejima, H.; Asaka, T., Adaptation effects in static postural control by providing simultaneous visual feedback of center of pressure and center of gravity. Journal of physiological anthropology. 2017, 36, 31.

16. Yim, J.; Petrofsky, J.; Lee, H., Correlation between Mechanical Properties of the Ankle Muscles and Postural Sway during the Menstrual Cycle. The Tohoku journal of experimental medicine. 2018, 244, 201-207.

17. Eils, E.; Behrens, S.; Mers, O.; Thorwesten, L.; Völker, K.; Rosenbaum, D. J. G.; posture, Reduced plantar sensation causes a cautious walking pattern. 2004, 20, 54-60. 
18. Park, K. H.; Lim, J. Y.; Kim, T. H., The effects of ankle strategy exercises on unstable surfaces on dynamic balance and changes in the COP. Journal of physical therapy science. 2016, 28, 456-9.

19. Moraes, R.; Lopes, A. G.; Barela, J. A., Monocular vision and increased distance reducing the effects of visual manipulation on body sway. Neuroscience letters. 2009, 460, 209-13.

20. Kannape, O. A.; Barré, A.; Aminian, K.; Blanke, O., Cognitive loading affects motor awareness and movement kinematics but not locomotor trajectories during goal-directed walking in a virtual reality environment. PloS one. 2014, 9, e85560.

21. Mochizuki, G.; Semmler, J. G.; Ivanova, T. D.; Garland, S. J., Low-frequency common modulation of soleus motor unit discharge is enhanced during postural control in humans. Experimental brain research. 2006, 175, 584-95.

22. Nam, S. M.; Kim, K.; Lee, D. Y., Effects of visual feedback balance training on the balance and ankle instability in adult men with functional ankle instability. Journal of physical therapy science. 2018, 30, 113-115.

23. Nam, S.-m.; Kim, W.-b.; Yun, C.-k. J. J. o. p. t. s., Effects of balance training by knee joint motions on muscle activity in adult men with functional ankle instability. 2016, 28, 1629-1632.

24. Brown, C. N.; Ko, J.; Rosen, A. B.; Hsieh, K., Individuals with both perceived ankle instability and mechanical laxity demonstrate dynamic postural stability deficits. Clinical biomechanics (Bristol, Avon). 2015, 30, 1170-4.

25. Rougier, P., Visual feedback induces opposite effects on elementary centre of gravity and centre of pressure minus centre of gravity motions in undisturbed upright stance. Clinical biomechanics (Bristol, Avon). 2003, 18, 341-9.

26. Brauer, S. G.; Woollacott, M.; Shumway-Cook, A., The interacting effects of cognitive demand and recovery of postural stability in balance-impaired elderly persons. J. Gerontol. A Biol. Sci. Med. Sci. 2001, 56, M489-M496.

27. Nitz, J. C.; Kuys, S.; Isles, R.; Fu, S., Is the Wii Fit a new-generation tool for improving balance, health and well-being? A pilot study. Climacteric : the journal of the International Menopause Society. 2010, 13, 487-91.

28. DG, S.; MI, A.; K, M.; $\quad A H, \quad$.; $\quad H, O$.; $M R, P_{.} ; K, \quad$., Positive effect of balance training with visual feedback on standing balance abilities in people with incomplete spinal cord injury. Spinal cord. 2010, 48, 886-93.

29. Hsu, W.-L.; Scholz, J. P.; Schoner, G.; Jeka, J. J.; Kiemel, T., Control and estimation of posture during quiet stance depends on multijoint coordination. Journal of neurophysiology. 2007, 97, 3024-3035. 